Condensed Matter Physics, 2004, Vol. 7, No. 2(38), pp. 401-420

\title{
Band structure of non-steiochiometric large-sized nanocrystallites
}

\author{
I.V.Kityk* \\ Institute of Materials Science and Metallurgy, Polish Academy of Science. \\ Ul. Reymonta, 25, Cracow, Poland
}

Received December 8, 2004, in final form April 26, 2004

\begin{abstract}
A band structure of large-sized (from 20 to $35 \mathrm{~nm}$ ) non-steichiometric nanocrystallites $(\mathrm{NC})$ of the $\mathrm{Si}_{2-x} \mathrm{C}_{x}(1.04<x<1.10)$ has been investigated using different band energy approaches and a modified Car-Parinello molecular dynamics structure optimization of the NC interfaces. The nonsteichiometric excess of carbon favors the appearance of a thin prevailingly carbon-contained layer (with thickness of about $1 \mathrm{~nm}$ ) covering the crystallites. As a consequence, one can observe a substantial structure reconstruction of boundary SiC crystalline layers. The numerical modeling has shown that these $\mathrm{NC}$ can be considered as $\mathrm{SiC}$ reconstructed crystalline films with thickness of about $2 \mathrm{~nm}$ covering the SiC crystallites. The observed data are considered within the different one-electron band structure methods. It was shown that the nano-sized carbon sheet plays a key role in a modified band structure. Independent manifestation of the important role played by the reconstructed confined layers is due to the experimentally discovered excitonic-like resonances. Low-temperature absorption measurements confirm the existence of sharp-like absorption resonances originating from the reconstructed layers.
\end{abstract}

Key words: pseudopotential, band structure, nanocrystallites

PACS: 71.20

\section{Introduction}

Recently, there appeared a new direction in the nano-technology consisting in the possibility of using the nanocrystallites as materials for optics and electronics due to the size-dependent electronic and optical properties of these materials [1-8]. The so-called large-size nanocrystallites (NC) (larger than $10 \mathrm{~nm}$ ) are of particular interest. They demonstrate properties typical of the bulk crystalline materials as well as nano-sized effects originating from $\mathrm{SiC}$ reconstructed $\mathrm{NC}$ layers similar to quantized nano-dot structures. One can expect that the latter will be directly dependent on relative sizes of particular crystallites and the corresponding interface

*E-mail: i.kityk@wsp.czest.pl; kityk@torus.uck.pk.edu.pl 
environment. Moreover, one can expect the occurrence of excitonic interactions. Coexistence of bulk-like long-range ordered and quantum size-confined layers opens a rare possibility to operate by electron energy dispersion within the same crystallites contrary to the thin microcrystalline films deposited on crystalline surfaces [8].

Among many NC materials, the SiC crystallites were chosen for the modelling, since the technology of their production to obtain desired sizes was well developed. An energy gap of the $\mathrm{SiC}$ can vary within wide spectral ranges (from 2 to $4.5 \mathrm{eV}$ ), depending on a polytype structure. Furthermore, $\mathrm{SiC}$ is substantially more stable towards external mechanical and thermal treatments compared to other semiconductors.

The main goal of the present work is to model and simulate the effect of the $\mathrm{NC}$ sizes and carbon excess on the optical absorption of the large sized $\mathrm{Si}_{2-x} \mathrm{C}_{x}$. Contributions of the reconstructed near-the-interface states as well as the excitonic resonances to the observed absorption spectra have been separately evaluated.

The nano-sized confined effects are usually studied for the NC with sizes below 8$10 \mathrm{~nm}$, where clear effects of quantization are observed. In such cases, k-space bulklike dispersion disappears and discrete excitonic-like levels appear in the energy gap. However, the quantum-confined interface possessing the bulk-like as well as the dot-like quantized excitonic properties can be of interest for the experimental optical phenomena. These features are caused by the reconstructed $\mathrm{SiC}$ surfaces with the thickness up to $2 \mathrm{~nm}$, separating the bulk-like crystallites and surrounding amorphous-like background.

Depending on a relative content of the quantum confined states in the total $\mathrm{NC}$, one can evaluate relative contributions of the reconstructed $\mathrm{SiC}$ near the grain boundary to the band energy (BE) dispersion as well as to the optical absorption (directly connected with the imaginary part of dielectric susceptibility).

In section 2, several technological details of the $\mathrm{Si}_{2-x} \mathrm{C}_{x}$ large-sized $\mathrm{NC}$ synthesis are presented. Monitoring the $\mathrm{Si}_{2-x} \mathrm{C}_{x} \mathrm{NC}$ structural and morphological features is achieved by the use of the TEM, NMR, Raman and IR spectroscopic methods. A calculation technique for the molecular dynamics interface structure optimization is described in section 3. Results of the band structure (BS) calculations performed by different calculation techniques and comparison with the experimental optical data are presented in section 4. Particular attention is devoted to simulation of optical absorption induced by the BS of NC reconstructed levels as well as to the corresponding excitonic effects.

\section{Sample features}

The $\mathrm{SiC}$ large-sized nanopowders were synthesized by a $\mathrm{CO}_{2}$ laser pyrolysis of silane and acetylene gaseous mixtures following a technical device described in [2]. 


\subsection{Specimen synthesis, structure and morphology characterizations}

The reactant fluxes monitor the $\mathrm{C} / \mathrm{Si}$ ratios or silicon-rich network in the outermost $\mathrm{Si}_{2-x} \mathrm{C}_{x}$ nanoparticle surfaces. The present work bears on two $\mathrm{SiC}$ batches with atomic carbon excess of about $4 \%(x=1.04)$ and $10 \%(x=1.10)$. The former was synthesized by a flow rate of about $602 \mathrm{~cm}^{3} / \mathrm{mn}$ for silane and $314 \mathrm{~cm}^{3} / \mathrm{mn}$ for acetylene.

The $\mathrm{Si}_{2-x} \mathrm{C}_{x}$ (with $x=1.10$ ) was sunthesized by a flow rate of about $120 \mathrm{~cm}^{3} / \mathrm{mn}$ and $66 \mathrm{~cm}^{3} / \mathrm{mn}$, for silane and acetylene, respectively. Chemical analyses of the asformed powders shows only a low oxygen contamination $(<3 \%$ weight $)$ through the formation of silicate. Annealing at $1200^{\circ} \mathrm{C}$ was performed under argon in a high temperature graphite furnace at $600^{\circ} \mathrm{C} / \mathrm{min}$ heating rate and a dwell time of about 1 hour. Such a treatment favors homogenization and densification of the powders without altering the average size of the nanoparticles (26 nm for $x=1.04)$ and (50 $\mathrm{nm}$ for $x=1.10$ ). All the forthcoming experimental investigations concern the nanopowders annealed at $1200^{\circ} \mathrm{C}$.

In TEM investigations for $x=1.10$ batch, the $\mathrm{NC}$ exhibit rounded shapes with sizes between $10 \mathrm{~nm}$ and $100 \mathrm{~nm}$ and mono-domain-like single crystals with stacking faults. The external particle surface is covered with a thin $(\approx 1 \mathrm{~nm})$ layer of carbon. The major $(\mathrm{SiC})$ phase, more or less coating the grain surface, is identified as a polyaromatic carbon.

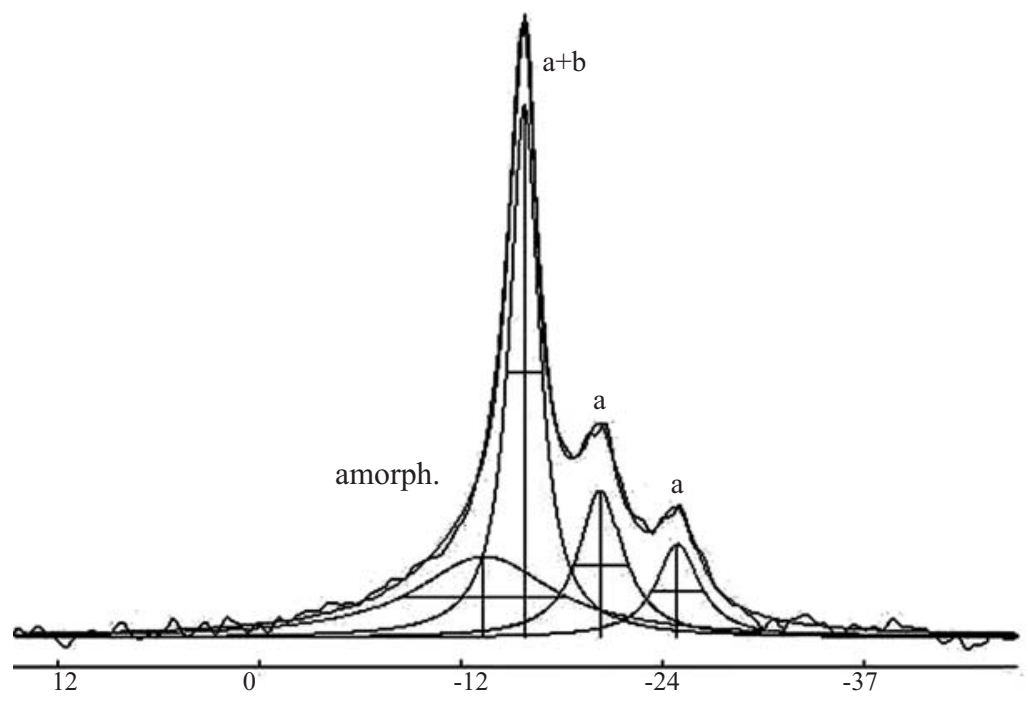

Figure 1. NMR spectrum of the $\mathrm{Si}_{0.96} \mathrm{C}_{1.04}$. The decomposition in several lines reflects coexisting structures $(6 \mathrm{H}-\mathrm{SiC}, 3 \mathrm{C}-\mathrm{SiC}$ and amorphous $\mathrm{SiC})$.

NMR investigations of short range were performed for $x=1.04$ specimens. The Si spectrum (figure 1) forms the polytypes involved in the sample. The triplet lines located at $-25,-20$ and $-16 \mathrm{ppm}$ with 1:1:2 relative ratios are associated with the $6 \mathrm{H}-\mathrm{SiC}$ structure. The single $-16 \mathrm{ppm}$ line is connected to $3 \mathrm{C}-\mathrm{SiC}$. The content of the $6 \mathrm{H}-\mathrm{SiC}$ is of about $26 \%$, whereas the $3 \mathrm{C}-\mathrm{SiC}$ has a relative concentration of $59 \%$. 
An amorphous structure is also involved with a relative contribution of about $12 \%$.

For $x=1.04$ batch, the powder is formed by agglomerated $\mathrm{SiC}$ grains with round shapes and a size ranging from 3 to $40 \mathrm{~nm}$. A layer of about $\approx 0.5 \mathrm{~nm}$ thickness covers the crystals and consists of amorphous silicon oxide or possibly silicon oxycarbon.

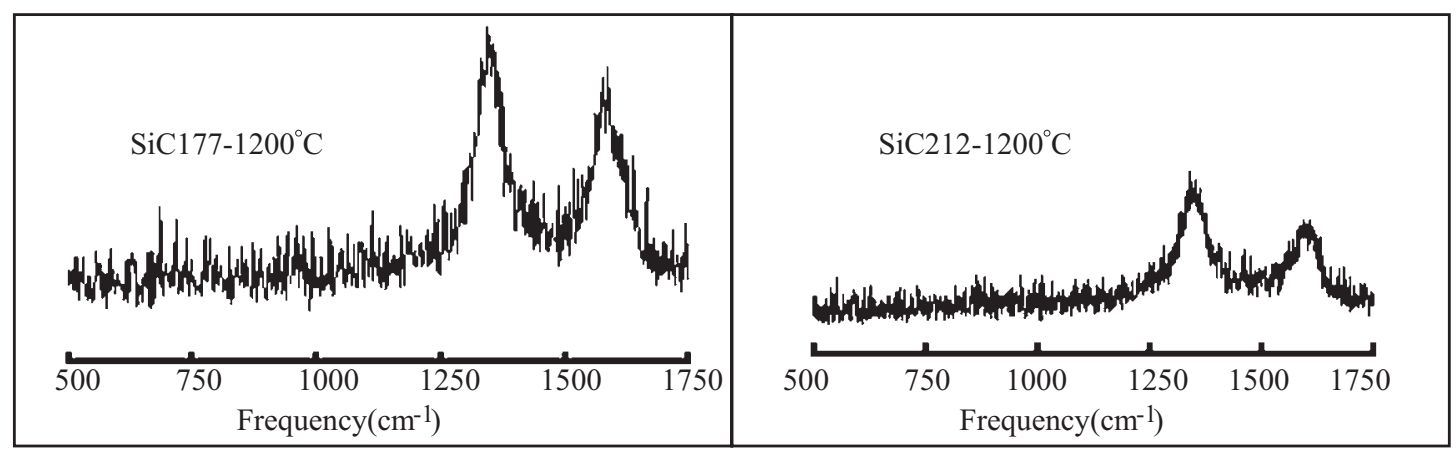

Figure 2. Raman spectra of the Si2-xCx with different $\mathrm{x}$ : (a) $x=1.10$; (b) $x=0.96$.

\subsection{Raman and IR investigations}

The Raman measurements were performed using an $\mathrm{Ar}^{+}$ion laser $(\lambda=488 \mathrm{~nm})$. The spectral width of about $4 \mathrm{~cm}^{-1}$ was a compromise between good resolution, sufficiently scattered light and low power absorbed by the sample $(3 \mathrm{~mW})$ in order to avoid the specimen overheating. The Raman spectra of the $\mathrm{Si}_{0.90} \mathrm{C}_{1.10}$ batches are presented in figure 2. These spectra demonstrate the presence of graphite with sp2 and sp3 bonding. The corresponding spectral bands located between 1347 and $1595 \mathrm{~cm}^{-1}$ originate from the in-plane $\mathrm{E}_{2 g}$ mode $\left(1595 \mathrm{~cm}^{-1}\right)$ and in-plane zoneboundary $\mathrm{A}_{1 g}$ mode $\left(1347 \mathrm{~cm}^{-1}\right)$. However, finite crystallite sizes or disorder in the Graphtec arrangement can lead to an active Raman of the $\mathrm{A}_{1 g}$ mode. Anyway, the Raman investigations are understandable through a rich carbon composition in external particle surfaces with high scattering efficiencies capable of screening the bulk particle mainly composed of SiC structures. No Raman signature from $\mathrm{SiC}$ was clearly detected by the Raman spectra. This means a large absorption coefficient of the surface composition.

The IR transmission spectrum of the $\mathrm{Si}_{0.96} \mathrm{C}_{1.04}$ sample incorporated in $\mathrm{KBr}$ platelet is shown in figure 3 . The main features consist in a characteristic $\mathrm{SiC}$ IR transmission band centered at $830 \mathrm{~cm}^{-1}$ and a weak band located at $1150 \mathrm{~cm}^{-1}$. The latter is accounted for by the Si-O stretching vibrations that occur in the $\mathrm{SiO}_{2}$. Similar features are observed in the IR spectrum of the $\mathrm{Si}_{0.90} \mathrm{C}_{1.10}$ sample where a complete coverage of the particle by thin carbon layer is realized. This permits to assertain the transparency of the $\mathrm{Si}_{2-x} \mathrm{C}_{x}$ in the IR range in contrast to the behavior in the visible range as developed above for Raman investigations. 


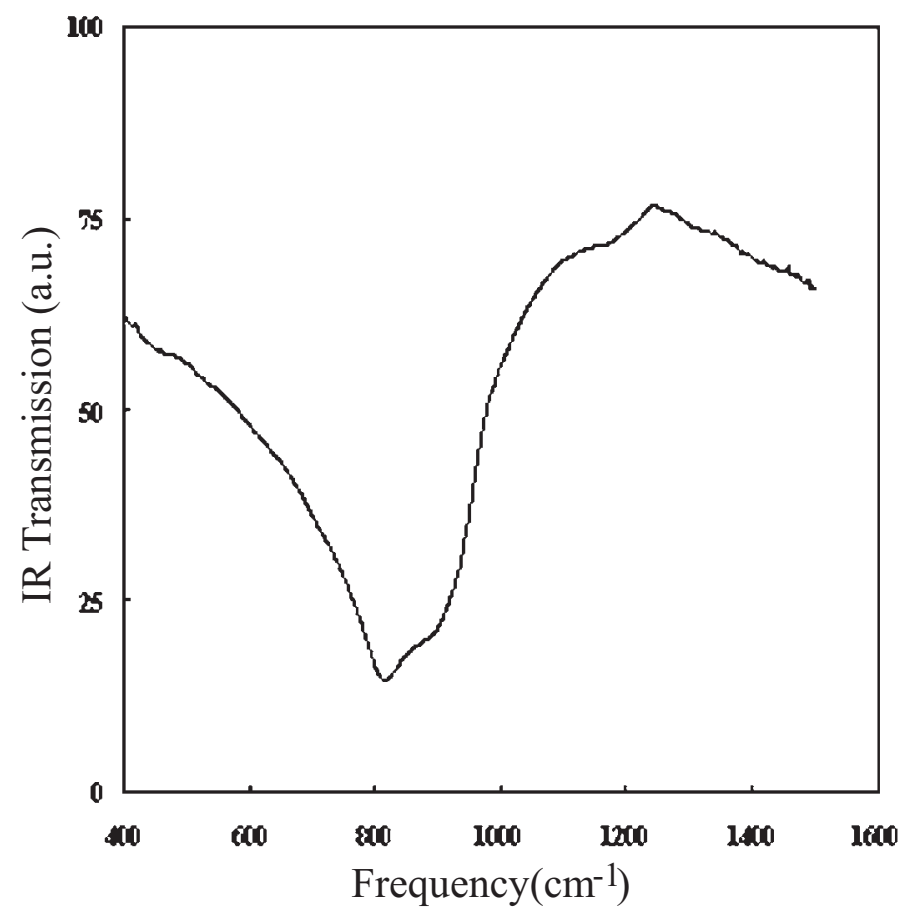

Figure 3. IR absorption spectra for the $\mathrm{Si}_{1.04} \mathrm{C}_{0.96}$ after oxidation.

\section{Molecular dynamics NC layer's structure optimization}

Silicon carbide occurs in several closely inter-related structural modifications that hardly differ one from another.

\subsection{Simulations of the carbon sheets}

All the structural modifications considered have tetrahedral bonds and their local arrangement is the same up to the second neighbors. All these structural types, expressed in terms of hexagonal units, have almost the same value of the lattice constant $a=5.825$ a.u., but a value of $\mathbf{c}$ depends on the polytype kind. Their structural difference may be understood by considering the stacking sequence of the hexagonal-like bi-layers (formed with pairs of $\mathrm{C}$ and $\mathrm{Si}$ layers). The atoms in each bi-layer have the following positions: $\alpha\left(0,0, \mathrm{c}_{1}\right) ; \beta\left(1 / 3,2 / 3, \mathrm{c}_{2}\right)$ and $\gamma\left(2 / 3,1 / 3, \mathrm{c}_{3}\right)$. At the same time, a vertical sequence of the bi-layers for the cubic polytypes are: $\alpha \beta \gamma \alpha \beta \gamma \mathfrak{u}$ These bi-layers have cubic-like as well as hexagonal-like site-symmetries. Due to the dominant role played by the hexagonal-like structural component, the effective hexagonal-like (wurtzite) lattice and corresponding Brillouine zone (BZ) will be considered here to be the basic ones during the BE calculations. Ratios of different structural fragments $(\alpha-\mathrm{SiC}$ and $\beta$-SiC $)$ were assumed when determining the starting structural factors used for the BE calculations and were determined from the NMR data (see figure 1). 


\subsection{Covering carbon sheet}

The structure and coordination bonding of carbon sheet covering the SiC nanoparticles were optimized using molecular dynamics (MD) calculations. Reconstructions with the carbon $s p$-bonded chains are energetically favored compared with the sp3like $\mathrm{C}$ bonds. The energy difference between two successive reconstruction geometry differs only by a few $\mathrm{meV} /$ dimmer. Moreover, the formation energy of sp3-like disturbances on a $s p$-bonded interface rapidly decreases with the increasing defect concentration. These lead to $s p \rightarrow s p 3$ transition on the crystallite surfaces.

Recent experiments [9] have independently confirmed that stable sp3-like bonded carbon lines can be formed on the C-terminated $\mathrm{SiC}\langle 001\rangle$ surface, by inducing an irreversible $s p \rightarrow s p 3$ phase transition in the substrate. These $s p 3$ bonds are possible precursors for the diamond growth on $\mathrm{SiC}$ surfaces.

Self-consistent calculations $[7,8]$ using the DFT approach within the local-density approximation (LDA) have shown that the most probable reconstruction of the Cterminated surface of cubic $\mathrm{SiC}\langle 001\rangle$ is a carbon possessing sp3-like carbon chains. Independently, experimental investigations [10] have shown that hydrocarbon evaporation on Si-terminated substrates yields $s p$-bonded carbon atoms, usually referred to as bridge reconstruction. The stability of $s p 3$ - and $s p$-bonded reconstruction, as inferred from first principles calculations using DFT and gradient corrected exchange energy functional was determined by the so-called generalized-gradient approximations (GGA) [11]. Possible coexistence of the $s p 2 \rightarrow s p 3$ transition observed experimentally was analyzed and has shown little probability.

\subsection{Molecular dynamics simulations of reconstructed SiC layers}

The geometry optimization of the reconstructed $\mathrm{Si}_{2-x} \mathrm{C}_{x}$ surfaces was started from the interface between the $\mathrm{NC}$ and carbon sheet background (calculation details are similar to the described by Albercht et.al., [12] (see figure 4).

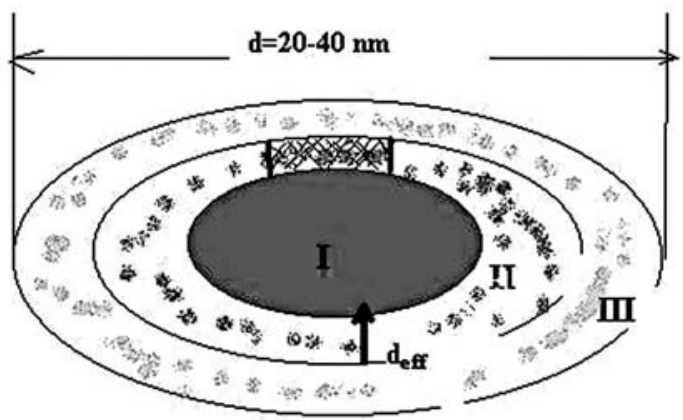

Figure 4. Principal schema of the grain obtained from the TEM data: I - crystallite phases; II - interface region; III - amorphous-like region.

The TEM and other structural data indicate that the investigated NC are formed by proper crystallites with a given $\alpha / \beta \mathrm{SiC}$ ratio. The TEM data show that nearthe-interface layers have thickness changing from 2 to $4 \mathrm{~nm}$. The EPR, X-ray and 
other structural investigations [2] have shown that the near-the-interface levels play a key role in the electronic properties of the $\mathrm{SiC} \mathrm{NC}$. It is also seen from the above mentioned figures that one chosen segment of the interface (squared in figure 4) is responsible for electronic properties of the whole composite. Sizes of the largesized NC will define only a partial contribution of the interface regions to the total electronic behavior.

The optimization was started from four neighboring layers: two layers from the crystallite side (indicated by I) and two layers in the direction of the carbon amorphous-like sheet background (indicated by II and III). 50-60 atoms from both sides of the interface have been taken into account. The MD procedure was carried out until the total energy minimum value for a given cluster was the same as for the whole cluster (re-normalized by one ion). At the second step, the next proper layers were considered and the procedure was repeated for the new total energy per ion. The iteration process was repeated until the relative ion displacement for two successive layers was less than $0.2 \AA$. The latter was found to be a limit in a precision when determining the atom position within the framework of the adopted model. More details concerning structural optimizations of the interfaces are given in the subsequent sections.

Afterwards, the structure of the surrounding (near-the-interface $\mathrm{SiC}$ ) sheets has been additionally optimized using the Car-Parrinello ab initio molecular dynamics (CPMD) [13] within a DFT pseudopotential description. The functional used in the CPMD was of the BLYP type $[14,15]$. The atom core positions and the PW expansion coefficients were treated as simultaneous dynamic variables. Simulations in this approach were performed using super-cells containing 128 atoms with a PW cutoff of 37 Ry. Periodic boundary conditions and $\Gamma$ point sampling of the Brillouin zone were used. The calculated total energies using $\Gamma$ point were found to be accurate to better than $0.05 \mathrm{eV}$ per atom. Martins-Trouiller pseudopotentials ([16]) in the Kleinmann-Bylander form were used to represent the carbon ion cores. This pseudopotential was checked for accuracy against the well-known molecules (benzene, methane, acetylene) possessing different carbon configurations.

At a PW cutoff of 40 Ry, the computed bond lengths fell within $2 \%$ of the observed experimental values. The so-called "liquid quench" method was employed to produce the final network at each density $\mathrm{C}$ configuration. The relative stability of the reconstructed interfaces was studied in order to address the controversy between the experiment and the existing DFT-LDA calculations. Previous evaluations have shown that the LDA approach underestimates the $\mathrm{SiC}$ bulk lattice constant by $1.69 \%$ and the pseudopotential approximation overestimates it by $0.33 \%$. The more preferable geometry corresponds to the lattice constant of about $4.312 \AA$.

\subsection{Optimization results of the reconstructed NC layer structure}

The performed calculations have shown that the near-the-interface $\mathrm{SiC}$ region (II in the figure 4) may be considered to be the reconstructed $\mathrm{SiC}$ quantum confined surfaces deposited on the SiC. The evaluated structural data are presented in table 1. Since they are dependent on the distance from $\mathrm{SiC}$ layer-crystalline interface, 
Table 1. Changes of effective structural parameters of the $\mathrm{SiC}$ reconstructed surfaces while moving from the amorphous-like level (region II-I) (carbon layerSiC crystallite) towards the deeper crystalline layers (III).

\begin{tabular}{|l|l|l|l|}
\hline $\begin{array}{l}\text { Distance } d_{\text {eff from the }} \text { carbon - SiC interface } \\
\text { in the direction of the } \\
\text { crystallite states [nm] }\end{array}$ & $\begin{array}{l}\text { a }[\mathrm{nm}], \mathrm{SiC}-3 \mathrm{C} \\
\text { cubic structural } \\
\text { component) }\end{array}$ & $\begin{array}{l}\text { a }[\mathrm{nm}], \mathrm{SiC}-6 \mathrm{H} \\
\text { (hexagonal struc- } \\
\text { tural component) }\end{array}$ & $\begin{array}{l}c / p \text { (hexago- } \\
\text { nal structural } \\
\text { component) }\end{array}$ \\
\hline 0.2 & $4.231(3)$ & $2.889(4)$ & $2.515(2)$ \\
\hline 0.4 & $4.256(4)$ & $2.916(3)$ & $2.515(1)$ \\
\hline 0.6 & $4.286(8)$ & $2.937(1)$ & $2.515(6)$ \\
\hline 0.8 & $4.309(1)$ & $2.953(5)$ & $2.514(4)$ \\
\hline 1.0 & $4.321(5)$ & $2.974(4)$ & $2.514(2)$ \\
\hline 1.2 & $4.326(7)$ & $2.989(2)$ & $2.514(3)$ \\
\hline 1.4 & $4.331(3)$ & $3.036(4)$ & $2.514(9)$ \\
\hline 1.6 & $4.346(1)$ & $3.068(1)$ & $2.513(3)$ \\
\hline 1.8 & $4.352(3)$ & $3.071(3)$ & $2.513(1)$ \\
\hline 2.0 & $4.359(4)$ & $3.072(1)$ & $2.513(0)$ \\
\hline
\end{tabular}

appropriate results are presented versus the distance d.

One can see that $d_{\text {eff }}$ shows a considerable increase of lattice parameters while moving from the carbon sheet towards deeper $\mathrm{SiC}$ layers. For the $\mathrm{d}_{\text {eff }}$ larger than $2 \mathrm{~nm}$, the reconstruction of the structure disappears and the bulk-like crystallite structure begins to dominate. It is striking that for the hexagonal structural component the $c / p$ parameters are more stable comparing with the a ones. The presented data show that the thickness of interface region is equal to about $2 \mathrm{~nm}$ which is an additional confirmation of the good agreement between the experimental and theoretical data.

Simulated by the above mentioned method, differential local charge density distributions versus the $\mathrm{d}_{\text {eff }}$ distance are presented in figure 5 . One can clearly notice a substantial charge density redistribution determining the electronic structure of the reconstructed surfaces. Glans et al., in 2000 independently demonstrated that the $\mathrm{SiC}$ epilayers deposited on the SiC-3C surfaces show a clear interface reconstruction. Since the sizes of the $\mathrm{SiC}$ crystallites are relatively large (of about $20 \mathrm{~nm}$ ), a model similar to the $\mathrm{SiC}$ epilayers deposited on the $\mathrm{SiC}$ crystallites can be proposed.

\section{Band energy structure calculations}

\subsection{Choice criterion for calculation technique}

Based on the structural data for the NC layered interfaces, one can expect the appearance of the BS with a substantially modified k-dispersion. Due to the decreasing effective lattice constants, the existence of a flatter band energy dispersion 

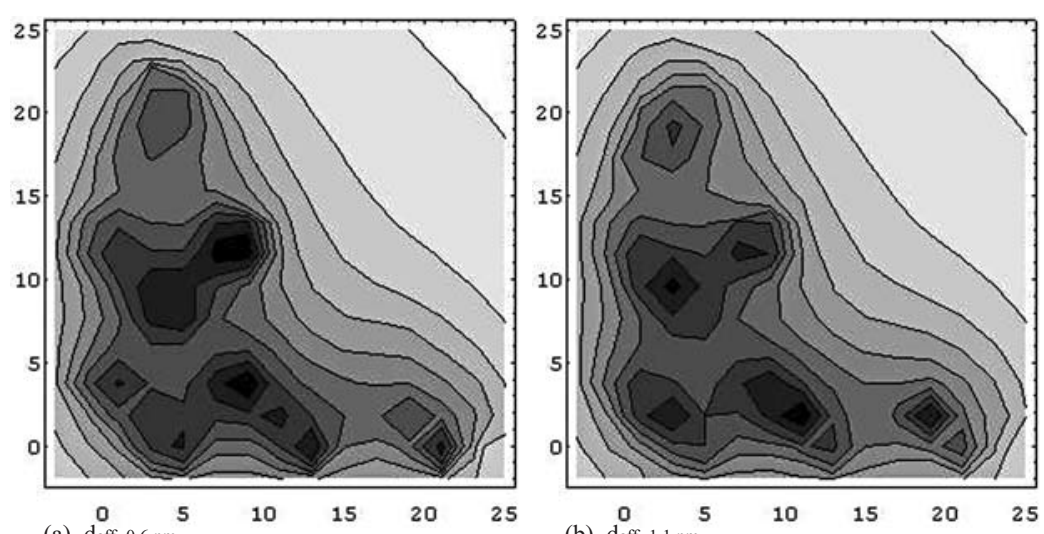

(a) deff $=0.6 \mathrm{~nm}$

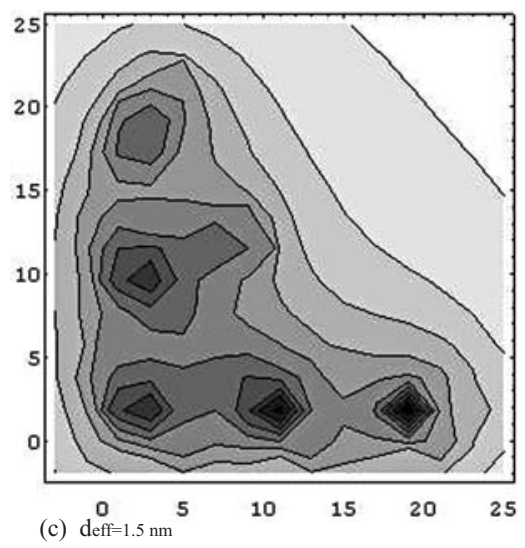

Figure 5. Differential charge density of the interface depending on the distance d from the interfaces.

and an increasing effective energy gap value can be expected.

There exist several works devoted to the BS calculations of the perfect bulk SiC crystals $([17,18])$. A choice of the BS calculation technique depends on the kind of properties we would like to simulate. Because we are interested in simulations of the optical features, a main criterion consists in maximal coincidence of the experimental optical parameters with the simulated ones. Therefore, we apply several band energy approaches, such as a self-consistent full linear augmented plane wave approach within the local density approximation (FLAPW-LDA) ([19]), a modified normconserving pseudopotential (NCPP) ([20]), a semi-empirical pseudopotential method (SEPM) ([21]). Among the BS calculation techniques, these methods seem to be more appropriate today in order to interpret the optical data. We will thus perform a comparison of the obtained theoretical data with the experimental ones.

\subsubsection{Norm-conserving pseudopotential method}

The total energy of crystalline system is expressed within a local density functional approximation (LDFA) with respect to the charge density $\rho(r)$. The nuclearelectron functional potential $\mathrm{V}_{n-e}[\rho(r)]$ is chosen to be in the form described by 
BACHELET, 1982. During the calculations there were included screened potentials $\mathrm{V}_{e-e}[\rho(r)]$ and $\mathrm{V}_{e-c}[\rho(r)]$, corresponding to Coulomb contribution and exchangecorrelation energy, respectively.

A nonlinear extrapolation procedure was carried out in order to calculate the fitting coefficients of the corresponding pseudo-wave-functions as well as of corresponding derivatives, in a form convenient for analytic evaluations of secular equation matrix elements, in particular :

$$
\psi(l, r, \beta)=\sum_{n} a_{n}^{\beta} r^{n} \exp \left[-a_{n}^{(l, \beta)} r^{n}\right]
$$

where $\beta$ denotes the atom type; $l$ is a corresponding angular momentum; $n$ determines a level of precision of the nonlinear fitting procedure and can vary from 1 to 5 . The $\mathrm{a}^{\beta}$ and $\alpha_{n}^{(l, \beta)}$ coefficients are the fitting coefficients evaluated during the nonlinear fitting procedure with a precision of the fit less than $0.03 \mathrm{eV}$.

The atomic pseudopotential was chosen in non-local (l-dependent) form:

$$
V_{\mathrm{ps}}^{(l, \beta)}=\sum_{i=1}^{3} A_{i}^{\beta}+r^{2} A_{i+3}^{\beta} \exp \left[-a_{l}^{(l, \beta)} r^{2}\right],
$$

where $\mathrm{A}_{i} \mathrm{~A}_{i+3}, \alpha_{1}^{(l, \beta)}$ are the fitting coefficients. The calculated atomic potentials were used as starting ones in forming the solid-state form-factors.

When we go from the atoms to a long-range plane wave basis set, we do the Fourier transformation from the real r-space up to the reciprocal k-space. The obtained Fourier transformers served as basic for the secular equation. The secular equation was solved appropriately to a pseudopotential expressed by equation 2 :

$$
\left\|\left[h^{2} \frac{\left(k+G_{n}\right)^{2}}{2 m}-E(k)\right] \delta_{n, n^{\prime}}+\sum_{\beta} V_{\beta}\left(G_{n}^{\prime}-G_{n}\right) S_{\beta}\left(G_{n}^{\prime}-G_{n}\right)\right\|=0
$$

where $E(k)$ is an eigenenergy for the k-point in the Brillouin zone, $G_{n}^{\prime}, G_{n}$ are wavevectors of the interacting basis plane waves. A structural form-factors for $\beta^{\text {th }}$ type atoms can be expressed as follows:

$$
S_{\beta}\left(G_{n}^{\prime}-G_{n}\right)=\frac{g^{(\beta)}}{\Omega N_{a} \sum \exp \left[-l\left(G_{n}^{\prime}-G_{n}\right) \tau_{\beta}\right]} .
$$

The positions of the $\beta^{\text {th }}$ atoms are determined from the above described molecular dynamics procedure.

Here $\mathrm{g}^{(\beta)}$ is a weighting factor determining a partial contribution to the total potential of a given structural component (for example $\alpha / \beta$-SiC ratio) or of the reconstructed layer presented above. A similar approach for different structural fragments has been successfully applied for binary solid alloys, glasses and organic materials $([23])$. One can expect that this approach would be also appropriate for different disordered and partially ordered solids including the investigated $\mathrm{Si}_{x} \mathrm{C}_{1-x}$ 
NC. Hexagonal-like BZ was chosen as a basic one because the wurtzite-like structure is more important (see section 2).

Therefore, the $\mathrm{g}^{(\beta)}$ weighting factors (determining the atom positions of the reconstructed surfaces) were introduced into appropriate structural factors corresponding to the contributions of different crystal subsystems. As a consequence, the reconstructed interface contribution is directly taken into account within the mentioned approach.

Electron screening effects were taken into account during the calculations of the total potential (7) using a parameterized Perdew-Zunger ([24]) approach. A special Chadhi-Cohen point method was applied in calculating the spatial electron charge density distribution. A diagonalization procedure was carried out at special weighting points of the BZ for each structural type.

Acceleration of the iteration convergence was achieved by transferring $75 \%$ of the $(m-1)^{\text {th }}$ iteration result to the $m^{\text {th }}$ iteration. The following condition was taken as a criterion for self-consistency:

$$
\left|\frac{\left(\rho_{m}-\rho_{m-1}\right)}{\rho_{m}}\right|<\varepsilon .
$$

During the calculations, a level of calculation error $(\varepsilon)$ was smaller than $0.10 \%$. To verify the dependence of $\mathrm{BE}$ calculation results on the choice of the $\alpha-\mathrm{SiC} / \beta-\mathrm{SiC}$ ratio, additional calculations were done for the perfect $\alpha$-SiC and $\beta$-SiC single crystals. The level of agreement with previously performed calculations of the electron density of states using LMTO methods was equal to about $0.3 \mathrm{eV}$. However, the main drawback of all one-electron LDF-LDSA calculations consists in underestimation of the band gap values. For this reason, a self-energy correction renormalization was carried out.

A basic BZ wurtzite-like hexagonal structure was chosen because it corresponds to the major structural component of the investigated NC. While varying structural factors obtained from the structural data and the MD geometry optimization, appropriate changes were introduced in equation (6).

In order to take into account the core-like state contributions, the norm-conserving PP wavefunctions were additionally modified through their orthogonalization to the LCAO wavefunctions. As a result, the total energy deviation from the energy cutoff and the Perdew-Alder screening parameter was stabilized within $0.22 \mathrm{eV}$.

\subsubsection{Local-density-derived semi-empirical pseudopotentials (SEPM)}

As a second method in calculating the semiconductor quantum structures, we have applied a modified semi-empirical pseudopotential (SEPM) approach introduced in [21]. This approach is proposed explicitly for the quantum dots, wires, wells and films with typical linear dimensions of 2-10 nm. A description of the electronic properties of such cluster structures using first-principles methods pertinent to bulk solids $([25])$ is currently prohibitive. The SEPM approach is much faster than the self-consistent first-principles methods used in the case of the bulk solids. 
Indeed, the Schrodinger equation is solved and an efficient diagonalization method providing energy levels in a fixed "energy window" is available and appropriate. Unlike effective-mass-based approaches ([17]), this method uses explicit and variationally flexible basis functions, permitting a direct comparison of the wave functions with local density approximation LDA studies when available. However, contrary to the LDA approach, the current method provides only electronic structure information, transition probabilities, wave functions but not ground-state properties such as equilibrium geometries, which have to be assumed at the outset.

The general mathematical formalism is similar to that described in section 4.2.1. In a scalar local-density approximation (SLDA), a scalar-relativistic atomic pseudopotential is obtained using the Troullier-Martins ([16]) procedure in order to perform a core-like correction. A plane-wave basis with a kinetic energy cutoff of 36.5 Ry was used. The non-local part of the ionic pseudopotential $V$ was obtained numerically. It was kept unchanged while moving from the LDA to the SLDA. The "small box" implementation was used to handle the nonlocal part of pseudopotential. This approach takes an advantage of the short-range nature of the nonlocal part and uses the plane waves (PW) basis set with large momentum to expand it in the Fourier space.

It is well known that the LDA band gaps are usually underestimated. Therefore, it is adjusted to reproduce the experimentally observed BE structures. The changes in the SLDA potentials are small comparing with the LDA results. The performed calculations have shown that sufficient sizes of the secular matrix varied from 170 to 240 .

Since the final pseudopotential was rather smooth, a rapidly converged PW expansion was possible. In fact, using the efficient diagonalization method and the full semi-empirical pseudopotential, the electronic structure for 90 atoms of the $\mathrm{SiC}$ supercluster was calculated.

\subsubsection{FLAPW-LDA method}

Among different BE calculation methods, the augmented plane wave (APW) methods were effectively developed. The well-known WIEN97 ${ }^{30}$ version is the full linear augmented plane wave local density approximation (FLAPW-LDA) and, in accordance with ([19]), the exchange-correlation Hedin-Lundquist ([26]) parameterization was performed. The $\mathrm{SiC}$ PW basis set was taken for the energy cutoffs of about $15 \mathrm{eV}$ and potential representation of about $52 \mathrm{eV}$. The expansion by angulardependent spherical harmonics was done for $l<10$. Approximately 16 states have been taken into account including 6 empty ones with a criterion of the self-consistent eigenenergy value stabilization of about $0.016 \mathrm{eV}$. Summation within the BZ was performed at 8 special points of the BZ. All the core states $(1 s C, 1 s S i$, 2sSi, $2 p S i)$ were recalculated during all iteration steps. A Thomas-Fermi screening potential was applied at the starting step and afterwards it was replaced by the Engel screened functions ([26]). Additional corrections were done using the generalized gradient approximation (GGA) to prevent energy gap underestimation.

The plane wave basis set was limited by wave vectors smaller than $9 / R_{m}$ ( where 


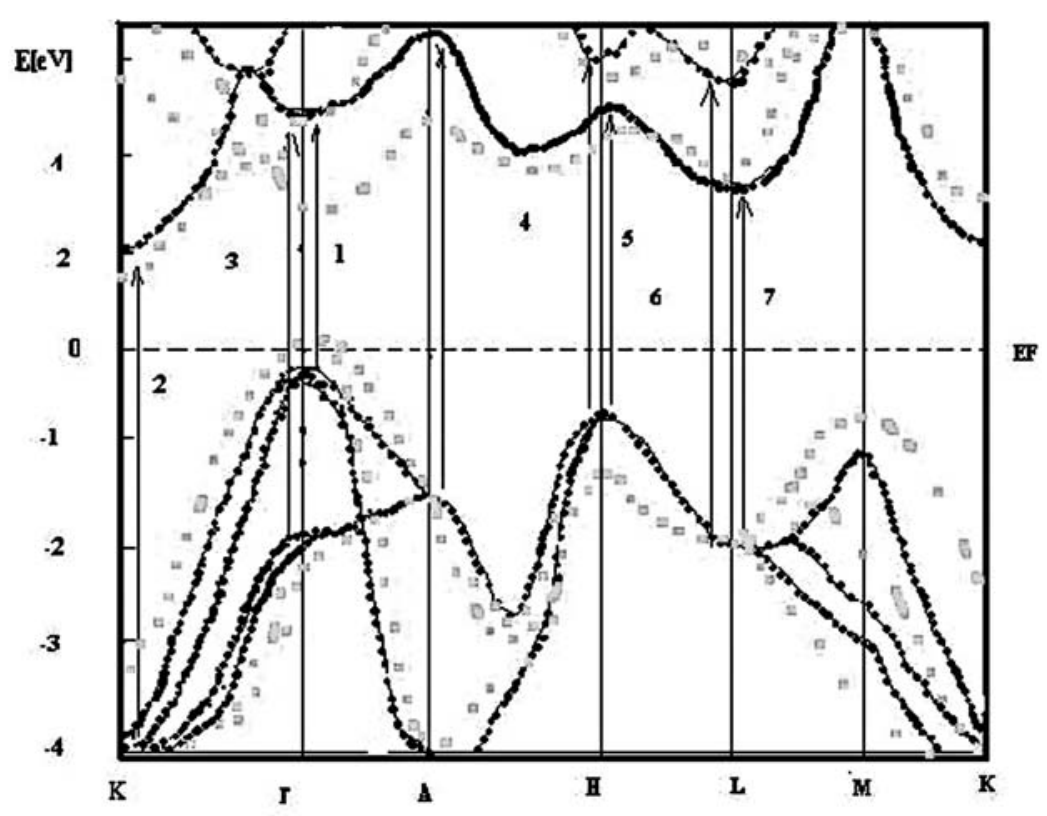

Figure 6. Fragment of the BE structure of the $\mathrm{SiC}$ calculated with inclusion of the near-the-interface inclusion projected into the hexagonal-like structure calculated by the modified NCPP method. The points indicate the reconstructed BE points.

$\mathrm{R}_{\mathrm{m}}$ is a muffin-tin sphere radius). The $4 \mathrm{H}$ structure requires 1340 plane waves and $3 \mathrm{C}$-structure needs 312 ones. The radius of the Si sphere was varied within the range $R_{\mathrm{Si}}=(1.08-1.16) R_{\mathrm{C}}$. The interacting spheres were chosen as non-overlapping (just touching) ones. The $1 s \mathrm{C}$ and 1,2s Si wavefunctions were taken as core states. All remaining orbitals were considered to be valence states orbitals.

For numerical calculations of the total and partial density of states (DOS), numerical simulations were conducted by means of a tetrahedron method using $96 \mathrm{k}-$ points for the $\beta$-polytype and $122 \mathrm{k}$-points for the $\alpha$-polytype with energy resolution of $0.12 \mathrm{eV}$. The considered atoms are relatively light and the spin-orbit interaction was not necessary to be included in the considerations. Accounting for the scalarrelativistic part leads to the compulsory inclusion of corresponding perturbations. In classifying the BZ, the notions presented in [9] were used.

Typical fragments of the $\mathrm{BE}$ dispersions calculated for the hexagonal-like $6 \mathrm{H}$ wurtzite structure modified by near-the-interface structure are shown in figure 6 and figure 7 . The presentation is limited only by the NCPP as well as by the SEPM scheme because the FLAPW data give the results much further from the experimental data compared with the mentioned ones. One can observe the appearance of substantial deviations from the perfect BE structure. It is worth emphasizing that within the SEPM scheme a considerable deviation is observed not only for the valence states, but for the conduction band states as well. This fact confirms the structural reconstruction for the near-the-interface state and the drawbacks of the semi-empirical method in describing the excited states. For comparison, a corresponding behavior achieved for the cubic fragment using the FLAPW-LDA approach 


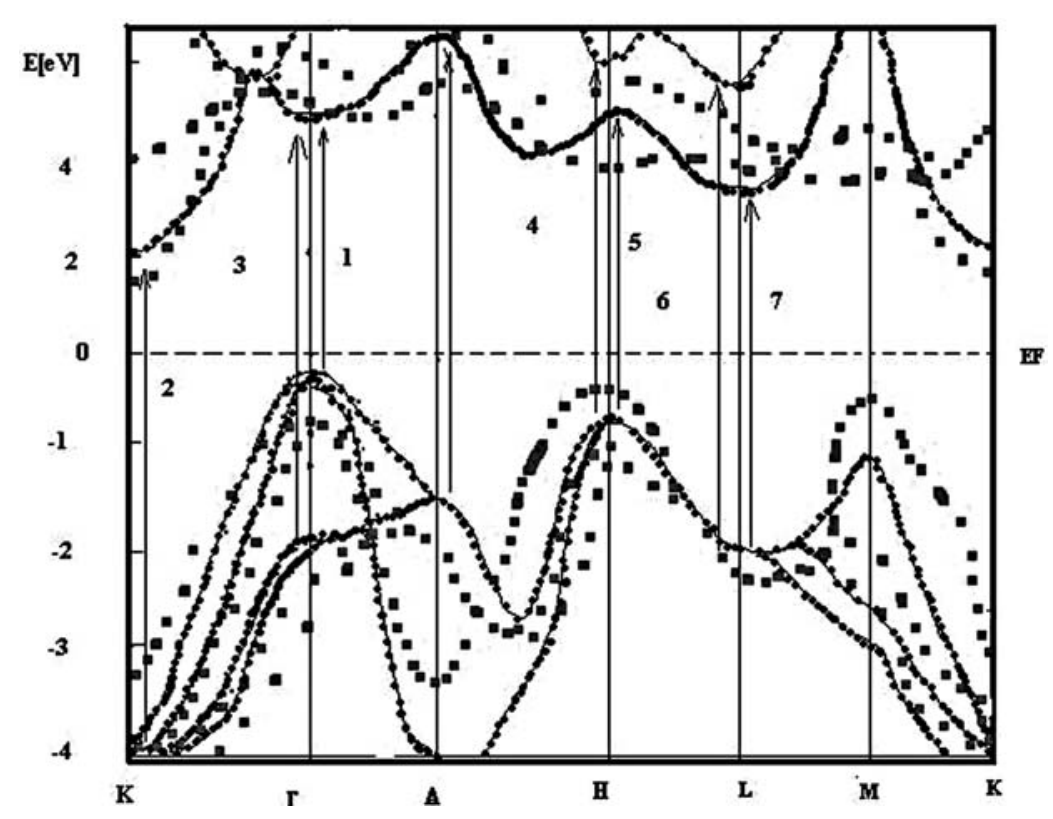

(a)

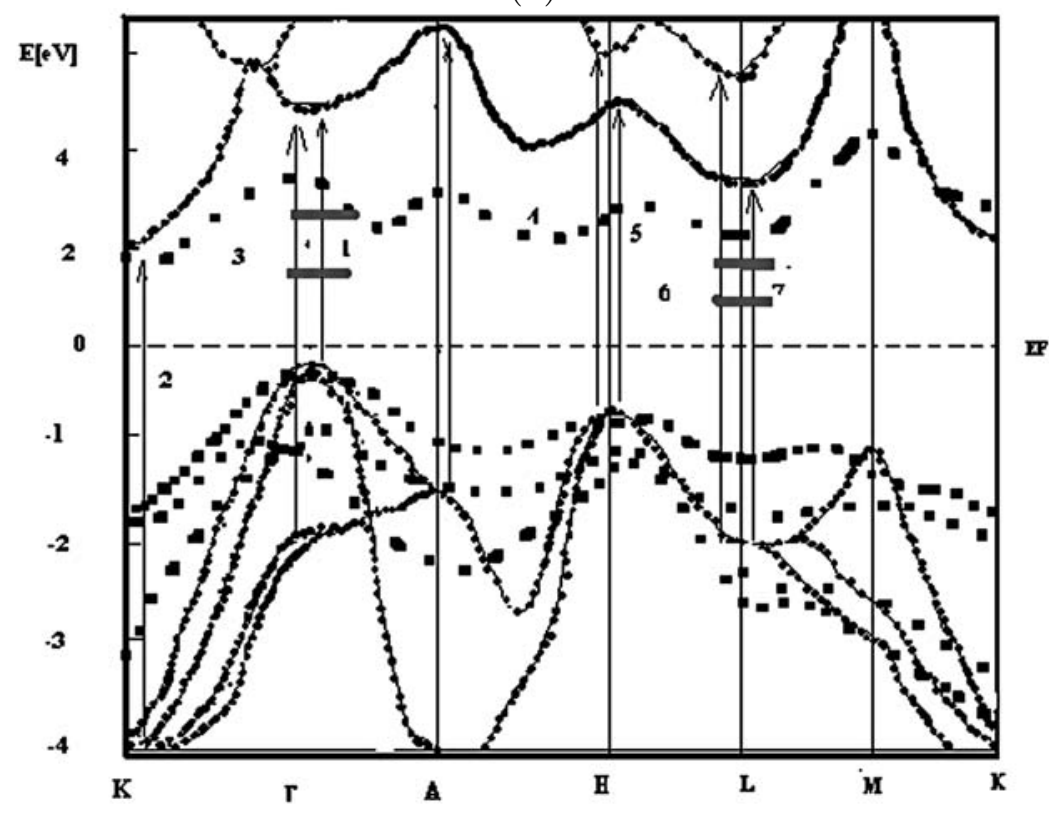

(b)

Figure 7. (a) Fragment of the BE structure of the SiC calculated by the SEPM approach with inclusion of the near-the-interface inclusion projected into the hexagonal-like structure. The points indicate the reconstructed BE points. (b) Fragment of the reconstructed BE structure modified by the reconstructed surface calculated within the FLAPW-LDA approach. The projection is done to the cubic-like structure. 


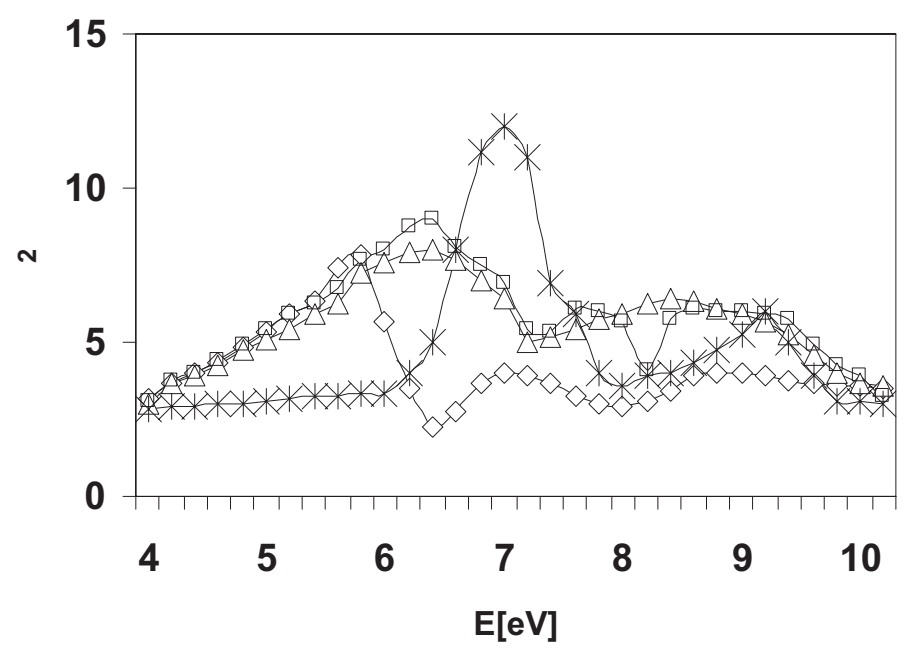

Figure 8. Spectral dependence of the e2(E) measured by ellipsometry and evaluated using different approaches: solid line - FLAPW method, dotted line modified NCPP, dashed line - SEPM. Experimental points are taken from [27]

is presented in figure 8. One can notice that the deviation is very large in this case. The latter may reflect a substantial presence of the non-cubic structural phase.

As an important criterion of the applicability of a given method, the structural (see table 2) and the BE parameters (see table 3) can be compared. It is obvious that the maximal agreement between the experimental energy gap and the theoretically calculated optical data and electronic structure is given in the case of the modified NCPP approach. The SEPM and FLAPW methods lead to results being substantially different from the experimental data.

\subsection{Optical absorption}

In order to simulate the optical properties, calculations of the imaginary part of dielectric susceptibility optical tensors were done using an expression:

$$
\begin{aligned}
\varepsilon_{2}(E)=\frac{2 \pi N e^{2}}{h^{3}} \sum_{v . b . k} \int & \mathrm{H} \mid\left\langle\Psi \Psi_{v . b} .(k, r)\left|\nabla_{r} \Psi \Psi_{c . b .}(k, r)\right|\right\rangle \\
& \times d^{3} r \delta\left(E_{v . b}(k) .-E_{c . b}(k) .-E\right),
\end{aligned}
$$

where $\Psi \Psi_{v . b} .(\mathbf{k}, \mathbf{r})$ and $\Psi \Psi_{c . b} \cdot(\mathbf{k}, \mathbf{r})$ corresponds to the valence and conduction band pseudowavefunction, respectively. All the summations should be carried out over the whole BZ.

The general expression was introduced with accounting for the matrix dipole moments. The matrix dipole moments were evaluated using commutation of a space and single-particle Hamiltonian. An additionally performed numerical verification has shown that the nonlocal effects change the average oscillator strength up to 
Table 2. Lattice constants for the $4 \mathrm{H}$ - and $3 \mathrm{C}$-polytypes calculated using different methods

\begin{tabular}{|l|l|l|}
\hline & $4 \mathrm{H}-\mathrm{SiC}$ & $3 \mathrm{C}-\mathrm{SiC}$ \\
\hline $\mathrm{a}[\mathrm{A}]$ & & \\
\hline NCPP modified as in the present work & 3.071 & 4.359 \\
\hline FLAPW-LDA & 3.067 & 4.341 \\
\hline SEPM & 3.053 & 4.336 \\
\hline $\mathrm{c} / \mathrm{p}[\mathrm{A}]$ & & \\
\hline NCPP modified as in the present work & 2.511 & \\
\hline FLAPW-LDA & 2.503 & \\
\hline SEPM & 2.489 & \\
\hline Experimental $[27]$ & $a=3.073 ; c / p=2.513$ & $a=4.360$ \\
\hline
\end{tabular}

Table 3. Principal band energy parameters of the SiC crystals.

\begin{tabular}{|l|l|l|}
\hline & 3C-SiC & 4H-SiC \\
\hline VB $[\mathrm{eV}]$ & (MNCPP-8.24; FLAPW- & (MNCPP-8.06; FLAPW- \\
& LDA-8.07; SEPM-7.97) & LDA-7.93; SEPM-7.89) \\
\hline $\mathrm{CB}[\mathrm{eV}]$ & $($ MNCPP-6.68; FLAPW- & (MNCPP-6.15; FLAPW- \\
& LDA-6.10; SEPM-6.06) & LDA-6.18; SEPM-6.67) \\
\hline $\mathrm{E}_{g}[\mathrm{eV}]$ & (MNCPP-3.024; FLAPW- & (MNCPP-3.63; FLAPW- \\
& LDA-2.894; SEPM-2.74) & LDA-3.37; SEPM-;3.24) \\
\hline Experimental & $8.28-V B ; 6.45-C B ;$ & $8.04-V B ; 6.23-\mathrm{CB} ;$ \\
& $\mathrm{E}_{g}=3.02 \mathrm{eV}$ & $\mathrm{E}_{g}=3.5 \mathrm{eV}$ \\
\hline
\end{tabular}

$12 \%$. A plasmon peak in the energy loss spectra is shifted towards higher energies up to $1.5-2 \mathrm{eV}$. As a consequence, all the calculations were done with the inclusion of the nonlocal contributions. The real parts of the dielectric tensors were evaluated using Kramers-Kronig dispersion relations. The reflectivity and energy loss functions were obtained from a Fresnel's formula.

A crucial point in the calculations of the $\varepsilon_{2}(\mathrm{E})$ was the k-space integration. A linear tetrahedron method for the $286(324,436) \mathbf{k}$ points in the $1 / 24$ irreducible part of the $\mathrm{BZ}$ for $2 \mathrm{H}(3 \mathrm{C}, 4 \mathrm{H}) \mathrm{SiC}$ was applied. In the present paper during the band summation, $24(56,82)$ conduction bands were included in order to achieve convergent results for the $2 \mathrm{H}(3 \mathrm{C}, 4 \mathrm{H})$ structures. This convergence is a crucial point in calculating the energy loss-electron spectra, since their main peaks occur at higher energies compared with the experimental ones [27]. To account for the excitation aspect not included in the DFT-LDA electronic structure, the theoretical curves were shifted to higher energies by a constant value of $0.66 \mathrm{eV}$. The important point is that the mean value of the scissor operator was smaller than the quasiparticle shifts calculated for various transitions. It nearly approaches half of the quasiparticle 
shift calculated for the indirect fundamental gaps. This fact seems to be a general one considering optical gaps in contrast to the case where these gaps are derived from photoemission and inverse photoemission. We believe that the reason for the reduction is a consequence of the electron-hole interaction (excitonic effects) omitted in the theoretical description but present in the experiment.

The calculated BE gaps were of the direct type (with the top of the valence band and the bottom of the conduction band situated at the same point of the BZ). Figure 8 presents spectral dependences of the imaginary part of dielectric susceptibility $\varepsilon_{2}(\mathrm{E})$ calculated by the three mentioned methods. The $\varepsilon_{2}(\mathrm{E})$ calculated within the orthogonalization NCPP method gives a better agreement with the experiment compared with the other two methods. This fact together with the better structural (see table 2) and energy gap (see table 3) parameters indicates the choice of the NCPP method in performing the calculations. The modified NCPP method is thus more preferable in simulating the optical and electronic properties of the $\mathrm{SiC}$.

To describe the excitonic-like near-the-interface confined SiC states, a microscopic expression for the nonlocal susceptibility was derived both in linear and nonlinear regimes, including a three-dimensional description of electronic quantum states and their electron-hole Coulomb interactions. The knowledge of the nonlocal susceptibility allows us to calculate the excitonic matrix dipole moments determining the optical absorption. Explicit calculations of the local linear response of the excitons confined in $\mathrm{SiC}$ reconstructed surfaces were reported. Significant interference effects in the interacting $\mathrm{SiC}$ reconstructed layers, electron-hole wave functions (particularly in the coupled nanostructures) turn out to be proved. The response of the $\mathrm{SiC}$ quantized dot-like structure is based on a microscopic description of electronic quantum states and their Coulomb interactions.

Absorption spectra simulations in the linear-response regime for the $\mathrm{SiC}$ structures with actual geometry and composition were calculated. The new features in excitonic spectra arise, particularly in the coupled nanostructures, owing to the interference effects in the interacting electron-hole wave functions. One can conclude that the Coulomb effects on the local spectra should be taken into account in order to correctly assign the experimental features.

The microscopic expression of the nonlocal susceptibility, including the Coulomb interactions between electrons and holes, is described within the space dispersion regime. It was revealed that the energy exciton levels are practically insensitive to the $\alpha / \beta$ ratio.

Let us note that there is no sign of spatially indirect transitions connecting an electron and a hole localized in different $\mathrm{SiC}$ sublayers. More substantial was the inclusion of the inter-electron Coulomb correlation. For the larger carbon sheet, the two main peaks, arising from direct transitions located in either SiC layers, are red-shifted by the exciton binding energy towards the high-energy continuum.

Table 4 compares the contribution of the reconstructed $\mathrm{SiC}$ surface to the additional absorption for $550 \mathrm{~nm}$ wavelength that dominates the absorption behavior compared with the crystalline ones. However, the main contribution to the light absorption is caused by the excitons originating from the near-the-interface states 
Table 4. The origin of absorption caused by different mechanisms.

\begin{tabular}{|l|l|}
\hline Structural fragment & Absorption value, $\mathrm{cm}^{-1}$ \\
\hline SiC crystalline contribution & $50-70$ \\
\hline Reconstructed BE state & $200-250$ \\
\hline Graphite-like layer & $820-850$ \\
\hline Excitonic state & $10^{3}-10^{4} \mathrm{~cm}^{-1}$ \\
\hline
\end{tabular}

possessing a giant absorption compared with the traditional BE structural reconstruction (see table 2 and table 3 ).

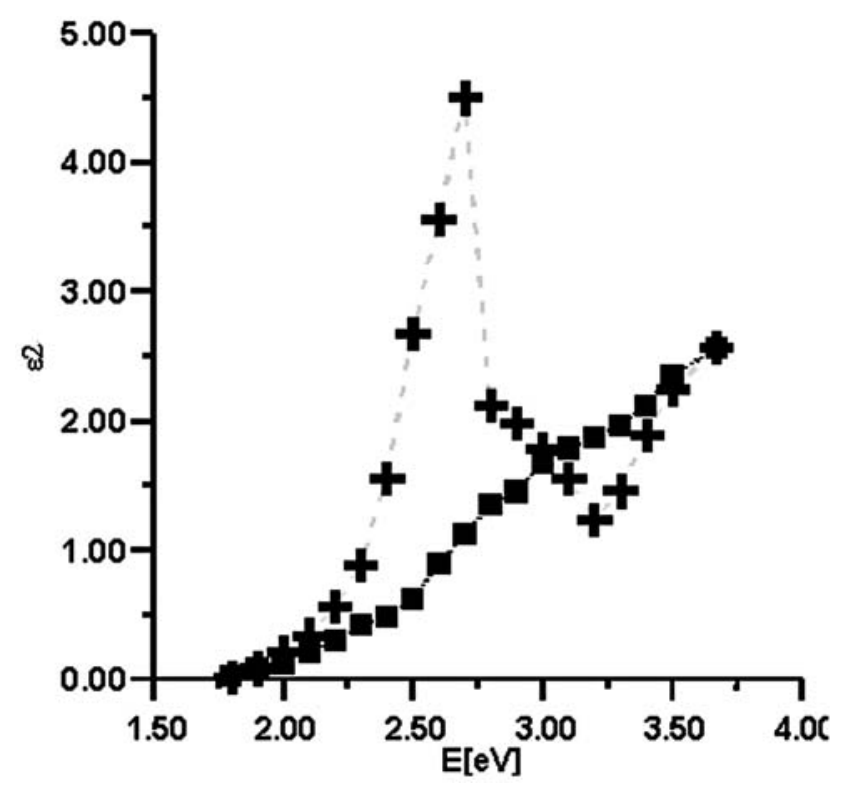

Figure 9. Absorption excitonic spectra of the $\mathrm{Si}_{2-x} \mathrm{C}_{x}$ at room (n) and helium liquid temperature (ם).

In order to verify the prediction about the appearance of the reconstructed layers, additional low-temperature measurements of absorption for the $\mathrm{Si}_{2-x} \mathrm{C}_{x}$ crystallites were done. Figure 9 presents two fragments of the spectra for two different temperatures together with the theoretically simulated excitonic-resonance absorption. One can notice that the exitonic absorption plays a major role in the absorption. This fact directly confirms the physical model considering the reconstructed surfaces as thin layers covering the crystallites.

The contribution of excitons leads to a substantial rearrangement of the remaining spectra and particularly to a substantial re-building of all the optical spectra. The data presented demonstrate that the dependence of the $\varepsilon_{2}(E)$ spectra versus the film thickness is vanishingly weak. Therefore, one can conclude that the main role in the features of excitonic-orginated $\varepsilon_{2}(E)$ spectra will be played by the reconstructed $\mathrm{SiC}$ surfaces and the thickness of the carbon sheet. This is in contradiction to the 
expectation from a reconstruction of the charge density distribution and reflects a relative stability of the interlayer interactions within a few surface layers.

\section{Conclusions}

A new model of describing the structural and BE properties of the large sized $\mathrm{Si}_{2-x} \mathrm{C}_{x} \mathrm{NC}$ is presented. The subsystem may be considered as reconstructed $\mathrm{SiC}$ crystalline layers (with thickness of about 2-4 nm) covering the $\mathrm{SiC}$ crystallites with the sizes 30-50 nm. The reconstructed $\mathrm{SiC}$ layers possess a flatter BE k-dispersion. Among the calculation methods, the best agreement with the experimental data is given by the norm-conserving non-local pseudopotential method together with the modified Car-Parinello MD simulations of interfaces. The varying reconstructed sheet thickness stimulates the appearance of disordered SiC crystallites NC crystalline state. Low-temperature experimental measurements confirm the dominant role of the excitonic resonances originated from the reconstructed layers. The excitonic-like states give a substantially higher contribution to the visible absorption compared with the reconstructed BE sub-band contributions. The reconstructed layers may be considered to be a new type of high performance electronic materials possessing quantum-confinement effects manifested in the excitonic spectra.

\section{Acknowledgements}

The author is grateful to CNRS - Region Rennes Delegation.

\section{References}

1. Kityk I.V., Makowska-Janusik M., Kassiba A., Plucinski K. // Optical Materials, 2000, No. 13, p. 449.

2. Charpentier S., Kassiba A., Bulou A., Monthhioux M., Cauhetier M. // Eur. Phys. J., AP, 1999, No. 8, p. 111.

3. Wang L.W., Zunger A. Nanocrystalline Semiconductor Materials (ed. by P.V.Kamat and D.Meisel). Amsterdam, Elsevier Science, 1996;

4. Yoffe D. // Adv. Phys., 1993, No. 42, p. 173.

5. Schlamp M.C., Peng X., Alivisatos A.P. // J. Appl. Phys., 1997, No. 82, p. 5837.

6. Buda F., Kohanoff J., Parrinello M. // Phys. Rev. Lett., 1992, No. 69, p. 1272.

7. Park Y.S. SiC Materials and Devices, Semiconductors and Semimetals. London, UK, Academic Press, 1998.

8. Yu M.B., Rusli S.F., Yoon S.F., Xu S.J., Chew K., Cui J., Ahn J., Zhang Q.

// Thin Solid Films, 2000, No. 377-378, p. 177.

9. Derycke V. // Phys. Rev. Lett., 1998, No. 81, p. 5868.

10. Bermudez V.M. // Phys. Stat. Solidi, 1997, No. B202, p. 447.

11. Gavrilenko V.I., Frolov S.I., Klyui N.I. // Physica, 1993, No. B185, p. 394.

12. Albercht S., Reining L., Del Sole R., Onida G. // Phys. Rev., 1999, No. B37, p. 7486.

13. Car R., Parinello M. // Phys. Rev. Lett., 1985, No. 55, p. 2471.

14. Becke A.D. // Phys. Rev., 1988, No. A38, p. 3098. 
15. Lee C., Yang W., Parr R.G. // Phys. Rev., 1988, No. B37, p. 785.

16. Troullier N., Martins J.L. // Phys. Rev., 1991, No. B43, p. 8861.

17. Persson C., Lindefelt U. // J. Appl. Phys., 1997, No. 82, p. 5496.

18. Adolph B., Tenelsen K., Gavrilenko V.I., Bechstedt F. // Phys. Rev., 1997, No. 55B, p. 1422 .

19. Asahi R., Mannstadt W., Freeman A.J. // Phys. Rev., 1999, No. 59B, p. 7486.

20. Kityk I.V., Kasperczyk J., Andrievskii B.V. // Phys. Letters, 1996, No. A216, p. 161.

21. Fu H., Zunger A. // Phys.Rev., 1997, No. B55, p. 1642.

22. Bachelet G.B., Hamann D.R., Schluter M. // Phys. Rev., 1982, No. B26, p. 4199.

23. Kityk I.V., Sahraoui B. // Phys. Rev., 1999, No. B60, p. 942.

24. Perdew J.B., Zunger A. // Phys. Rev., 1981, No. B23, p. 5048.

25. Dreizler R.M., Gross E.K. Density Functional Theory. Berlin, Springer Verlah, 1990.

26. Blochl P.E., Jepsen O., Andersen O.K. // Phys. Rev., 1994, No. B49, p. 16223.

27. Szao F., Wu B. // Chin. Acta Phys. Chem., 1999, No. 24, p. 412.

\title{
Зонна структура нестехіометричних широко-розмірних нанокристалів
}

\author{
I.В.Кітик \\ Інститут Матеріалів Науки та Металургії, Польська Академія Наук. \\ Вул. Реймонта, 25, Краків, Польща \\ Отримано 8 грудня 2003 р., в остаточному вигляді - 26 квітня \\ $2004 \mathrm{p}$.
}

Досліджено зонну структуру широко-розмірних $\mathrm{Si}_{2-x} \mathrm{C}_{x}(1.04<x<$ 1.10) нанокристалів (НК) з розмірами від 20 до 35 нм, використовуючи різні методи розрахунків зонної структури і модифікований молекулярно-динамічний метод геометричної оптимізації інтерфейсів Кара-Парінелло. Нестехіометричний надлишок вуглецю сприяє появі тонких (біля 1 нм) шарів вуглецю на поверхнях НК. В результаті спостерігається істотна перебудова граничних кристалічних шарів $\mathrm{SiC}$. Числове моделювання показало, що ці НК можуть розглядатись як модифіковані структури кристалів $\mathrm{SiC}$ з розмірами біля 2 нм. Отримані результати розглядаються в рамках різних одно-електронних підходів. Незалежним експериментальним проявом цих наноефектів $€$ екситонні ефекти, які проявляються в низькотемпературних спектрах поглинання як гострі абсорбційні резонанси, що походять реконструйованих шарів.

Ключові слова: псевдопотенціал, зонна струтура, нанокристаліти

PACS: 71.20 